\title{
RAPID MULTICOLOUR VARIATIONS OF SELECTED \\ SOUTHERN BE STARS *
}

\author{
S. ŠTEFL, P. HARMANEC \\ Astronomical Institute, Academy of Sciences of the Czech Republic, \\ CZ-25165 Ondrejov, Czech Republic \\ and \\ D. BAADE \\ European Southern Observatory, Karl-Schwarzschild-Str. 2, \\ D-85748 Garching bei München, Germany
}

\section{Introduction and observational database}

Many previous surveys of the rapid variability of Be stars (e.g., Balona et al. 1987, Cuypers et al. 1989, Balona et al 1992) were limited to the Strömgren $b$-band only. We have undertaken a new observational effort with the specific aim of studying colour variations. A search for new rapidly variable stars should provide us with statistical material needed to generalize properties of the phenomenon with respect to the stellar parameters. Some preliminary results of these items are presented here.

$u v b y$ and $\mathrm{H} \beta$ measurements were obtained in three observing runs at the European Southern Observatory with the Danish automatic $0.50-\mathrm{m}$ telescope in 1991 November, 1992 May/June, and 1992 December/1993 January. Additional photometric observations were secured from the South African Astronomical Observatory in parallel to the first two runs. Thirty stars covering all B spectral sub-classes were observed.

\section{Results}

The amplitudes of the variations usually stay below $0 r^{m} 12$ and do not differ much between the four passbands. But generally they are the largest in the $u$ band and decrease towards longer wavelengths. The variations in the $b-y$ and $m_{1}$ indices only in some cases reach a few hundredths of a magnitude but mostly remain within the scatter of the observations of the check stars $\left(0 a^{m} 01-0 r^{m} 02\right)$. By contrast, variations in the $u-b$ or $c_{1}$ indices are well detectable for $85 \%$ of the variable stars observed.

We can distinguish 3 types of variations which often occur in various combinations:

* Based on observations obtained at the European Southern Observatory, La Silla, Chile, and the South African Astronomical Observatory. 
1. (Quasi-) periodic variations with periods of the order of one day which, at least for early-B spectral sub-classes, are characteristic of Be stars.

2. Ephemeral brightness changes by up to $0{ }^{m} 2$ on a typical time scale of days. We observed both a drop and an increase in brightness. A decrease by $0 r^{m} 05$ in $u$ and $0 \cdot^{m} 02$ in $b$ accompanied by a reddening of $0 r^{m} 03$ in $u-b$ and no significant change in $b-y$ was observed for HD 98922. The event was almost symmetric in time, but the star was fainter by about $0 .^{m} 01$ after it returned to its quiescent phase. The pole-on star $\mu$ Cen became brighter by $0 r^{m} 26$ in $u$ and $0 r^{m} 16$ in $b$ during 4 days in January 1993. The star got bluer by $00^{m} 1$ in $u-b$ but redder by $0 r^{m} 05$ in $b-y$. The observed part of the event was nearly symmetric, too. It may correspond to the "outbursts" in $\mathrm{H} \alpha$ equivalent width reported for $\mu$ Cen by Baade et al. (1988) and Hanuschik et al. (1993).

3. Smooth medium-term variations on a time scale of up to tens of days and amplitudes of a few hundredths of a magnitude (e.g. DX Eri).

From a comparison of the type- 1 variabilities in $u-b$ and $u$, we find that most stars are bluer when brighter. Only for one-third of our sample such a relation is not well pronounced, and no star was found that showed the opposite trend.

No variations above the noise limit $\left(\approx 0 m^{m} 01\right)$ were detected in 5 of 7 program stars with spectral types later than $\mathrm{B} 7$, in agreement with previous photometric and spectroscopic searches (Balona et al. 1992, Baade 1989). The full amplitude of the B8-9 IIIe star HR 4221 was $0 r^{m} 025$. Balona et al. (1992) derived a period of $0.870 \mathrm{~d}$ for variations of the same amplitude observed in January 1988. The star HD 98922 (B9Ve), which showed the large-amplitude event described above, may be the most active known late B-type star if future observations prove the photospheric origin of its activity and thereby exclude a binary model.

In addition to HD 98922 also HR 4009 was identified as a new rapidly variable Be star. Our observations of HR 4009 span only 20 nights but indicate 2 candidate periods near $0.8 \mathrm{~d}$ in all four Strömgren bands and the $u-b$ and $c_{1}$ indices.

\section{References}

Baade D.: 1989, $A \& A$ 222, 200

Baade D., Dachs J.,v.d. Weygart R., Steeman F.: 1988, $A \& A$ 198, 211

Balona L.A., Marang F., Monderen P., Reiterman A., Zickgraf F.-J.: 1989, $A \& A S$ 71, 11

Balona L.A., Cuypers J., Marang F.: 1992, A\&AS 92, 533

Cuypers J., Balona L.A., Marang F.: 1989, $A \mathscr{E} A S$ 81, 151

Hanuschik R.W., Dachs J., Baudzus M., Thimm G.: 1993, $A \in A$ 274, 356 